\title{
Estimation of unknown function of a class of integral inequalities
}

\author{
Hua Zhong ${ }^{\mathrm{a}}$, Wu-Sheng Wang ${ }^{\mathrm{b}}$, Lele Fan ${ }^{\mathrm{c}^{*}}$ \\ School of Mathematics and Statistics, Hechi University, Guangxi, Yizhou 546300, P. R. China \\ aasonfan@163.com, bwang4896@126.com, ${ }^{\mathrm{c}}$ fanlele262@sohu.com
}

Keywords: Integral inequality; Integral inequality technique; Modified Riemann-Liouville fractional derivative; Riemann-Liouville fractional integral; Estimation of solution.

\begin{abstract}
It is well known that differential equations, integral equations and integral-differential equations have gained considerable importance and attention due to their applications in many engineering and scientific disciplines as the mathematical modeling of systems and processes in the fields of physical, mechanics, chemistry, aerodynamics, and the electrodynamics of complex mediums, etc. Gronwall-Bellman inequality is an important tool in the study of existence, uniqueness, boundedness, and other qualitative properties of solutions of differential equations and integral equation. In this paper, we discuss we establish a class of retarded iterated integral inequalities, which includes a nonconstant term outside the integrals. By integral inequality technique, the upper bound of the embedded unknown function is estimated explicitly. The derived result can be applied in the study of solutions of fractional integral equations.
\end{abstract}

\section{Introduction}

It is well known that differential equations and integral equations have gained considerable importance and attention due to their applications in many engineering and scientific disciplines as the mathematical modeling of systems and processes in the fields of physical, mechanics, chemistry, aerodynamics, and the electrodynamics of complex mediums, etc. In the study of the existence, uniqueness, boundedness, stability, continuous dependence on initial data and parameters of solutions, oscillation and other qualitative properties of solutions of differential equations and integral equations, one often deals with certain iterated integral inequalities. In 1919, Gronwall [1,2] introduced the famous Gronwall inequality, which can be stated as follows: If $u$ and $f$ are non-negative continuous functions on an interval $[a, b]$ satisfying

$$
u(t) \leq c+\int_{a}^{t} f(s) u(s) d s, t \in[a, b],
$$

for some constant $c \geq 0$, then

$$
u(t) \leq c \exp \left(\int_{a}^{t} f(s) d s\right), t \in[a, b] .
$$

In 2011, Abdeldaim et al. [3] studied a new integral inequality of Gronwall-Bellman-Pachpatte type

$$
u(t) \leq u_{0}+\int_{0}^{t} f(s) u(s)\left[u(s)+\int_{0}^{s} h(\tau)\left[u(\tau)+\int_{0}^{\tau} g(\xi) d \xi\right] d \tau\right] d s .
$$

In 2014, El-Owaidy, Abdeldaim, and El-Deeb [4] investigated a new retarded nonlinear integral inequality

$$
u(t) \leq f(t)+\int_{a}^{t} g(s) u^{p}(s) d s+\int_{a}^{\alpha(t)} h(s) u^{p}(s) d s .
$$

In 2014, Zheng [5] discussed the inequality of the following form

$$
u(t) \leq C+\frac{1}{\Gamma(\alpha)} \int_{0}^{t}(t-s)^{\alpha-1} g(s) u(s) d s+\frac{1}{\Gamma(\alpha)} \int_{0}^{T}(T-s)^{\alpha-1} g(s) u(s) d .
$$

In this paper, on the basis of $[3,4,6]$, we discuss a new iterated nonlinear integral inequality

$$
u(t) \leq f(t)+\frac{1}{\Gamma(\alpha)} \int_{0}^{t}(t-s)^{\alpha-1} g(s) u(s) d s+\frac{1}{\Gamma(\alpha)} \int_{0}^{t}(t-s)^{\alpha-1} g(s) u(s)[u(s)
$$




$$
\left.+\frac{1}{\Gamma(\alpha)} \int_{0}^{s}(s-\tau)^{\alpha-1} h(\tau) u(\tau) d \tau\right] d s
$$

\section{Result}

Throughout this paper, let $R_{+}=[0,+\infty)$.

The modified Riemann-Liouville derivative and fractional integral, presented by Jumarie in $[7,8]$, is defined by the following expression.

Definition 1. The modified Riemann-Liouville derivative of order $\alpha$ is defined by the following expression:

$$
D_{t}^{\alpha} f(t)=\left\{\begin{array}{l}
\frac{1}{\Gamma(1-\alpha)} \frac{d}{d t} \int_{0}^{t}(t-\xi)^{-\alpha}(f(\xi)-f(0)) d \xi, 0<\alpha<1, \\
\left(f^{(n)}(t)\right)^{(\alpha-n)}, n<\alpha<n+1, n \geq 1 .
\end{array}\right.
$$

Definition 2.The Riemann-Liouville fractional integral of order $\alpha$ on the interval is defined by

$$
I^{\alpha} f(t)=\frac{1}{\Gamma(1+\alpha)} \int_{0}^{t} f(s)(d s)^{\alpha}=\frac{1}{\Gamma(\alpha)} \int_{0}^{t}(t-s)^{\alpha-1} f(s) d s .
$$

In 2014, Zheng [5] proved the following property

Lemma 1. Suppose that $0<\alpha<1, \mathrm{f}$ is a continuous function, then

$$
D_{t}^{\alpha}\left(I^{\alpha} f(t)\right)=f(t) \text {. }
$$

Some important properties for the modified Riemann-Liouville derivative and fractional integral are listed as follows (see $[9,10])$.

$$
\begin{aligned}
& D_{t}^{\alpha} t^{r}=\frac{\Gamma(1+r)}{\Gamma(1+r-\alpha)} t^{r-\alpha}, \\
& D_{t}^{\alpha}(f(t) g(t))=g(t) D_{t}^{\alpha} f(t)+f(t) D_{t}^{\alpha} g(t) . \\
& D_{t}^{\alpha} f[g(t)]=f_{g}^{\prime}[g(t)] D_{t}^{\alpha} g(t)=D_{t}^{\alpha} f[g(t)]\left(g^{\prime}(t)\right)^{\alpha}, \\
& I^{\alpha}\left(D_{t}^{\alpha} f(t)\right)=f(t)-f(0), \\
& D_{t}^{\alpha} C=0, \text { where } C \text { is a constant. }
\end{aligned}
$$

Theorem 1. Suppose that $g(t), h(t) \in C\left(R_{+}, R_{+}\right), f \in\left(R_{+}, R_{+}\right)$is a nondecreasing function with $f(t)>$ 0 for $t>0$. Suppose that

$$
\left.1-\frac{1}{\Gamma(\alpha)} \int_{0}^{t}(t-\tau)^{\alpha-1} \exp \left[-\int_{0}^{\frac{\tau^{\alpha}}{\Gamma(1+\alpha)}}\left[g\left((s \Gamma(1+\alpha))^{\frac{1}{\alpha}}\right)+h\left((s \Gamma(1+\alpha))^{\frac{1}{\alpha}}\right)\right] d s\right] g(\tau) f(\tau) d \tau\right\}^{-1}>0 .
$$

If $u(t)$ satisfies (5), then

$$
\begin{aligned}
u(t) \leq & f(t) \exp \left[-\int_{0}^{\frac{t^{\alpha}}{\Gamma(1+\alpha)}}\left[g\left((s \Gamma(1+\alpha))^{\frac{1}{\alpha}}\right)+h\left((s \Gamma(1+\alpha)) \frac{1}{\alpha}\right)\right] d s\right]\{1 \\
& \left.\left.-\frac{1}{\Gamma(\alpha)} \int_{0}^{t}(t-\tau)^{\alpha-1} \exp \left[-\int_{0}^{\frac{\tau^{\alpha}}{\Gamma(1+\alpha)}}\left[g\left((s \Gamma(1+\alpha))^{\frac{1}{\alpha}}\right)+h\left((s \Gamma(1+\alpha)) \frac{1}{\alpha}\right)\right] d s\right] g(\tau) f(\tau) d \tau\right)\right\}^{-1} .
\end{aligned}
$$

Proof. Since $\mathrm{f}(\mathrm{t})$ is a positive and nondecreasing function. From (5) we obtain

$$
\begin{aligned}
\frac{u(t)}{f(t)} \leq & +\frac{1}{\Gamma(\alpha)} \int_{0}^{t}(t-s)^{\alpha-1} g(s) \frac{u(s)}{f(s)} d s+\frac{1}{\Gamma(\alpha)} \int_{0}^{t}(t-s)^{\alpha-1} g(s) f(s) \frac{u(s)}{f(s)}\left[\frac{u(s)}{f(s)}\right. \\
& \left.+\frac{1}{\Gamma(\alpha)} \int_{0}^{s}(s-\tau)^{\alpha-1} h(\tau) \frac{u(\tau)}{f(\tau)} d \tau\right] d s .
\end{aligned}
$$

Let $v(t)=u(t) / f(t)$. We have 


$$
\begin{aligned}
v(t) \leq 1 & +\frac{1}{\Gamma(\alpha)} \int_{0}^{t}(t-s)^{\alpha-1} g(s) v(s) d s+\frac{1}{\Gamma(\alpha)} \int_{0}^{t}(t-s)^{\alpha-1} g(s) f(s) v(s)[v(s) \\
& \left.+\frac{1}{\Gamma(\alpha)} \int_{0}^{s}(s-\tau)^{\alpha-1} h(\tau) v(\tau) d \tau\right] d s .
\end{aligned}
$$

Define a function $z(t)$ by the right hand side of the above inequality

$$
\begin{aligned}
z(t)=1 & +\frac{1}{\Gamma(\alpha)} \int_{0}^{t}(t-s)^{\alpha-1} g(s) v(s) d s+\frac{1}{\Gamma(\alpha)} \int_{0}^{t}(t-s)^{\alpha-1} g(s) f(s) v(s)[v(s) \\
& \left.+\frac{1}{\Gamma(\alpha)} \int_{0}^{s}(s-\tau)^{\alpha-1} h(\tau) v(\tau) d \tau\right] d s,
\end{aligned}
$$

which is a positive and nondecreasing function on $R_{+}$. From (17) and (18) we have

$$
\begin{aligned}
& v(t) \leq z(t), t \in R_{+}, \\
& z(0)=1 .
\end{aligned}
$$

By use of Lemma 1, using (19) we have

$$
\begin{aligned}
D_{t}^{\alpha} z(t) & =g(t) v(t)+g(t) f(t) v(t)\left\{v(t)+\frac{1}{\Gamma(\alpha)} \int_{0}^{t}(t-\tau)^{\alpha-1} h(\tau) v(\tau) d \tau\right\} \\
\leq & g(t) z(t)+g(t) f(t) z(t)\left\{z(t)+\frac{1}{\Gamma(\alpha)} \int_{0}^{t}(t-\tau)^{\alpha-1} h(\tau) z(\tau) d \tau\right\} \\
= & g(t) z(t)[1+f(t) w(t)],
\end{aligned}
$$

where

$$
w(t)=z(t)+\frac{1}{\Gamma(\alpha)} \int_{0}^{t}(t-\tau)^{\alpha-1} h(\tau) z(\tau) d \tau,
$$

which is a positive and nondecreasing function on $R_{+}$. From (20) and (22) we have

$$
\begin{aligned}
& z(t) \leq w(t), t \in R_{+}, \\
& w(0)=z(0)=1 .
\end{aligned}
$$

Using (21) and (23), we have

$$
\begin{aligned}
& D_{t}^{\alpha} w(t)=D_{t}^{\alpha} z(t)+h(t) z(t) \leq g(t) z(t)[1+f(t) w(t)]+h(t) z(t) \\
& \quad \leq g(t) w(t)[1+f(t) w(t)]+h(t) w(t) \leq g(t) f(t) w^{2}(t)+[g(t)+h(t)] w(t),
\end{aligned}
$$

Since $w(t)>0$, from $(25)$ we have

$$
D_{t}^{\alpha}\left(-(w(t))^{-1}\right) \leq[g(t)+h(t)](w(t))^{-1}+g(t) f(t) .
$$

Let $x(t)=-(w(t))^{-1}$, then $x(0)=-(w(0))^{-1}=-1$, from (26) we get

$$
D_{t}^{\alpha} x(t)-[g(t)+h(t)] x(t) \leq g(t) f(t) .
$$

On other hand, using the properties (9), (10), (11), we have

$$
\begin{aligned}
D_{t}^{\alpha}\left\{x(t) \exp \left[-\int_{0}^{\frac{t^{\alpha}}{\Gamma(1+\alpha)}}\left[g\left((s \Gamma(1+\alpha))^{\frac{1}{\alpha}}\right)+h\left((s \Gamma(1+\alpha))^{\frac{1}{\alpha}}\right)\right] d s\right]\right\} \\
=\exp \left[-\int_{0}^{\frac{t^{\alpha}}{\Gamma(1+\alpha)}}\left[g\left((s \Gamma(1+\alpha))^{\frac{1}{\alpha}}\right)+h\left((s \Gamma(1+\alpha))^{\frac{1}{\alpha}}\right)\right] d s\right] D_{t}^{\alpha} x(t) \\
\quad-x(t) \exp \left[-\int_{0}^{\frac{t^{\alpha}}{\Gamma(1+\alpha)}}\left[g\left((s \Gamma(1+\alpha))^{\frac{1}{\alpha}}\right)+h\left((s \Gamma(1+\alpha))^{\frac{1}{\alpha}}\right)\right] d s\right][g(t)+h(t)] D_{t}^{\alpha}\left(\frac{t^{\alpha}}{\Gamma(1+\alpha)}\right) \\
=\exp \left[-\int_{0}^{\frac{t^{\alpha}}{\Gamma(1+\alpha)}}\left[g\left((s \Gamma(1+\alpha))^{\frac{1}{\alpha}}\right)+h\left((s \Gamma(1+\alpha))^{\frac{1}{\alpha}}\right) d s\right]\left[D_{t}^{\alpha} x(t)-[g(t)+h(t)] x(t)\right]\right.
\end{aligned}
$$




$$
\leq \exp \left[-\int_{0}^{\frac{t^{\alpha}}{\Gamma(1+\alpha)}}\left[g\left((s \Gamma(1+\alpha))^{\frac{1}{\alpha}}\right)+h\left((s \Gamma(1+\alpha))^{\frac{1}{\alpha}}\right) d s\right] g(t) f(t)\right] .
$$

Substituting $t$ with $\tau$ in (28), making a fractional integral of order $\alpha$ for (28) with respect to $\tau$ from 0 to $t$ and using the properties (12), we obtain that

$$
\begin{aligned}
& x(t) \exp \left[-\int_{0}^{\frac{t^{\alpha}}{\Gamma(1+\alpha)}}\left[g\left((s \Gamma(1+\alpha))^{\frac{1}{\alpha}}\right)+h\left((s \Gamma(1+\alpha))^{\frac{1}{\alpha}}\right)\right] d s\right]-x(0) \\
& \leq \frac{1}{\Gamma(\alpha)} \int_{0}^{t}(t-\tau)^{\alpha-1} \exp \left[-\int_{0}^{\frac{t^{\alpha}}{\Gamma(1+\alpha)}}\left[g\left((s \Gamma(1+\alpha))^{\frac{1}{\alpha}}\right)+h\left((s \Gamma(1+\alpha))^{\frac{1}{\alpha}}\right)\right] d s\right] g(\tau) f(\tau) d \tau
\end{aligned}
$$

From (19), (23) and (29), we obtain the required estimation (15). The proof is complete.

\section{Summary}

In this paper, we discuss we establish a class of retarded iterated integral inequalities. Using several properties for the modified Riemann-Liouville derivative and fractional integral, the upper bound of the embedded unknown function is estimated explicitly.

\section{Acknowledgement}

This research was supported by Guangxi Natural Science Foundation (Project No. 2012GXNSFAA053009) and Scientific Research Foundation of the Education Department of Guangxi Autonomous Region of China (No. KY2015ZD103, KY2015LX340). The corresponding author is Lele Fan.

\section{References}

[1] T.H. Gronwall, Note on the derivatives with respect to a parameter of the solutions of a system of differential equations, Ann Math. 20 (1919)292-296.

[2] R. Bellman, The stability of solutions of linear differential equations, Duke Math J. 10 (1943) 643-647.

[3] A. Abdeldaim and M. Yakout, On some new integral inequalities of Gronwall-Bellman-Pachpatte type, Appl Math Comput. 217(2011)7887-7899.

[4] H. El-Owaidy, A. Abdeldaim and A. A. El-Deeb, On some new retarded nonlinear integral inequalities and theirs Applications, Mathematical Sciences Letters, 3(3)(2014)157-164.

[5] B. Zheng, Explicit bounds derived by some new inequalities and applications in fractional integral equations, Journal of Inequalities and Applications, 2014(4)(2014)1-12.

[6] B. G.Pachpatte, Inequalities for Differential and Integral Equations, London, Academic Press, 1998.

[7] G. Jumarie, Modified Riemann-Liouville derivative and fractional Taylor series of nondifferentiable functions further results, Comput. Math. Appl. 51(2006)1367-1376.

[8] G. Jumarie, Table of some basic fractional calculus formulae derived from a modified RiemannLiouville derivative for non-differentiable functions,Appl. Math. Lett. 22 (2009)378-385.

[9] GC . Wu, and EWM. Lee, Fractional variational iteration method and its application. Phys. Lett. A 374, (2010)2506-2509

[10] B. Zheng, (G'/G)-expansion method for solving fractional partial differential equations in the theory of mathematical physics. Commun. Theor. Phys. 58(2012)623-630. 